\title{
Interaction between LncRNA-ROR and miR-145 contributes to epithelial-mesenchymal transition of ovarian cancer cells
}

\author{
Jie $\mathrm{Li}^{1}$, Songlin Zhang ${ }^{2}$, Lei $\mathrm{Wu}^{3}$ and Meili $\mathrm{Pei}^{3}$ \\ ${ }^{1}$ Department of Pathology, The First Affiliated Hospital of Xian Jiaotong University, Xian, China \\ ${ }^{2}$ Department of Structural Heart Disease, the First Affiliated Hospital of Xian Jiaotong University, Xian, China \\ ${ }^{3}$ Department of Gynecology and Obstetrics, The First Affiliated Hospital of Xian Jiaotong University, Xian, China
}

\begin{abstract}
Emerging evidence has indicated that long noncoding RNAs (LncRNAs) play critical roles in tumor development. LncRNA-regulator of reprogramming (ROR) could enhance the malignance of numerous tumors through promoting cell reprogramming and the chemoresistance of several cancers. Nevertheless, the detailed molecular mechanisms of LncRNA-ROR in ovarian cancer are not well elucidated. In our study, we demonstrated that LncRNA-ROR was high expression in ovarian cancer tissues than in normal ovarian tissues, and LncRNA-ROR level was positively associated with clinical stages and the differentiation grades of malignant cells. Functionally, LncRNA-ROR could induce epithelial-mesenchymal transition (EMT), and regulated ovarian cancer cell migration and invasion by decreasing the expression of tumor suppressive miR-145 and its target gene FLNB. Moreover, the binding site for miR-145 within LncRNA-ROR contributed to the reciprocal negative regulation of LncRNA-ROR and miR-145. Taken together, LncRNA-ROR promoted EMT by the miR-145/FLNB regulatory axis in ovarian cancer, providing a potential therapeutic target for ovarian cancer.
\end{abstract}

Key words: LncRNA-ROR - miR-145 - FLNB - EMT - Ovarian cancer

\section{Introduction}

Ovarian cancer is one of the main gynecologic malignancies. Most patients are diagnosed with advanced ovarian cancer due to lack of typical signs and symptoms. Most of them are more likely to have invasion and peritoneal metastasis to adjacent organs. The five-year survival rate of patients with ovarian cancer is only $31 \%$ (Fidler 1978). Distant metastasis is a major cause of the poor survival of these patients (Rauh-Hain et al. 2016; Tripathi et al. 2018; Zhang et al. 2018). Epithelial-mesenchymal transition (EMT) acts as a crucial role in embryonic development (Thiery 2003), and it has more recently been implicated in promoting carcinoma invasion and metastasis (Wang et al. 2017). In the process of EMT, epithelial and mesenchymal markers change correspondingly, that is, the expression of

Correspondence to: Jie Li, Department of Pathology, The First Affiliated Hospital of Xi'an Jiaotong University, 277 West Yanta Road, Xi'an, Shaanxi 710061, China

E-mail:lijiexj64@126.com epithelial markers decreases while that of mesenchymal markers increases. Epithelial markers include E-cadherin, cytokeratin, laminin-1 and ZO-1, and mesenchymal markers include $\mathrm{N}$-cadherin, Vimentin and fibronectin. LncRNAs are RNA molecules over 200 nucleotides in length and lack an open reading frame. LncRNAs play an important roles in the regulation of multiple major biological processes impacting development, differentiation, metabolism and cancer progression (Whitehead et al. 2009; Wilusz et al. 2009; Guttman and Rinn 2012).

The regulator of reprogramming (ROR, LncRNA-ROR) is a $2.6 \mathrm{~kb}$ long RNA comprised of four exons (Loewer et al. 2010). Studies have shown that LncRNA-ROR is significantly up-regulated in induced pluripotent stem cells (iPSCs) (Loewer et al. 2010), human embryonic stem cell (hESC) (Cheng and Lin 2013), and multiple cancer cells (Jiang et al. 2016; Wang et al. 2016a, 2016b; Arunkumar et al. 2017; Chen et al. 2017; Li et al. 2017b, Liu et al. 2017, Qu et al. 2017, Zhang et al. 2017, Shang et al. 2018) including esophageal squamous, prostate cancer, breast cancer, pancreatic cancer, oral cancer. Research has 


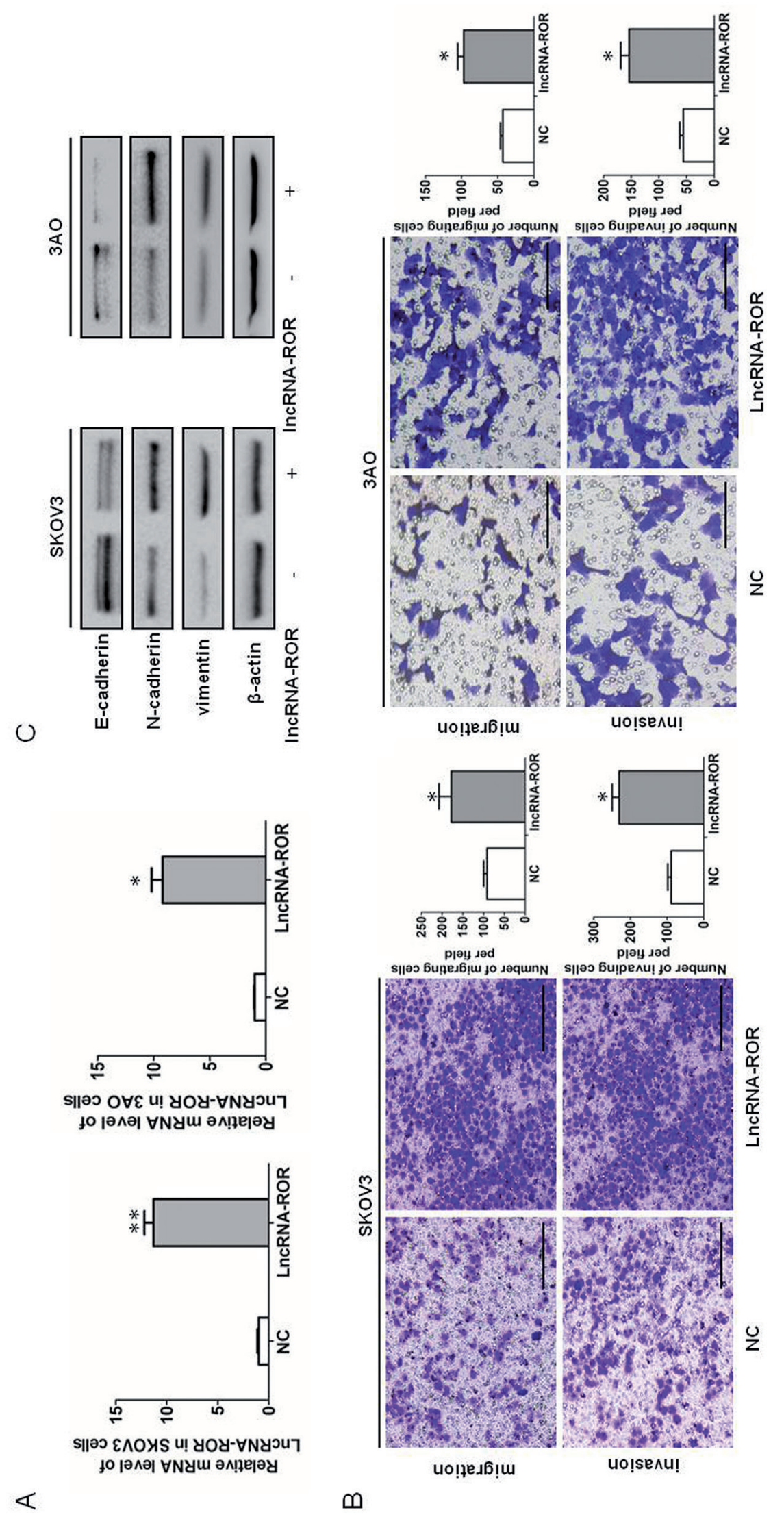




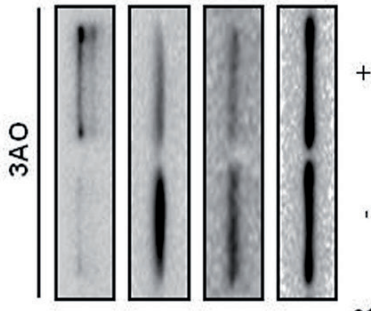

\section{$\frac{r}{0}$
$\frac{r}{i}$
$\frac{1}{6}$}
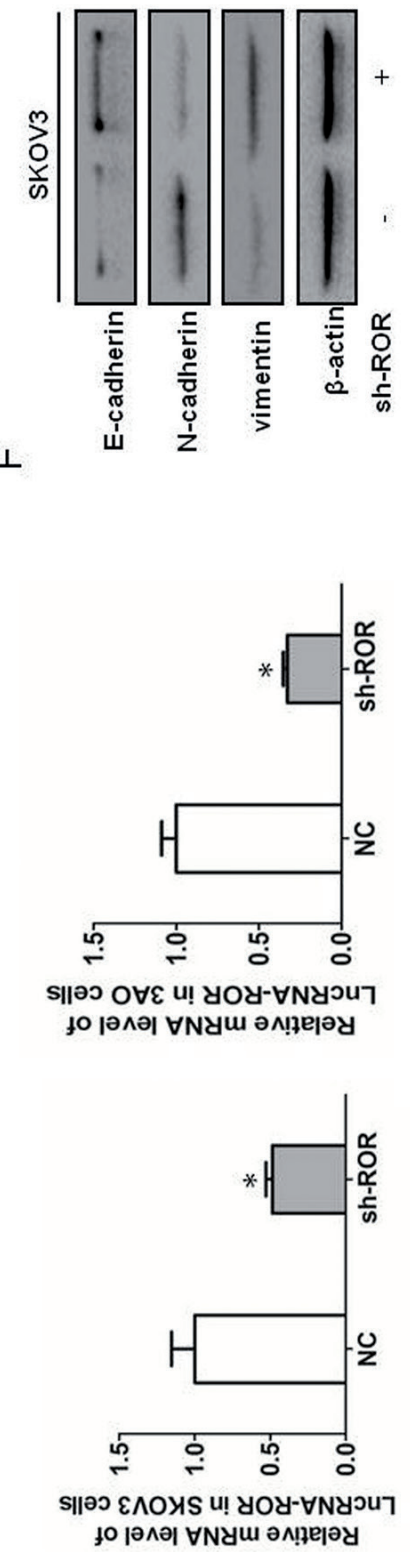

$\square$

ш

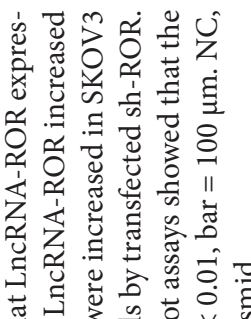

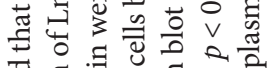

实

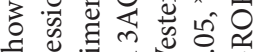

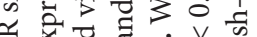

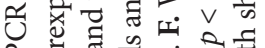

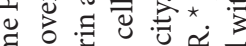

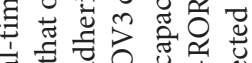

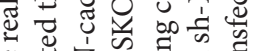

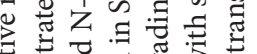

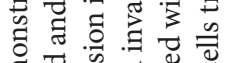

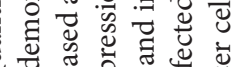

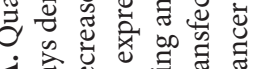

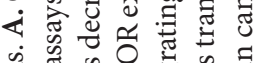

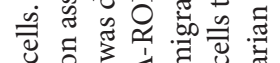

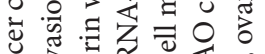

寻资出

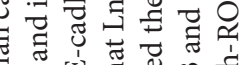

चี

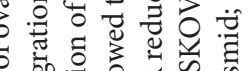

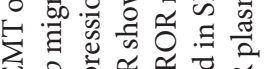

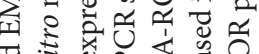

च :

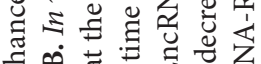

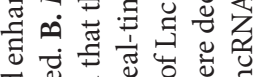

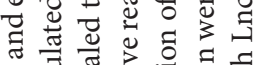

象

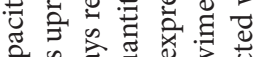

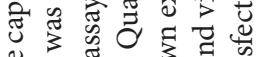

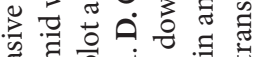

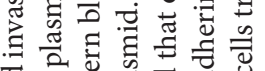

चै चु

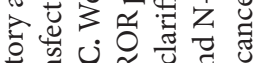

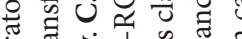

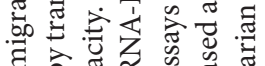

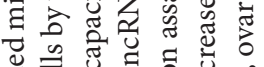

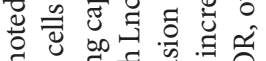

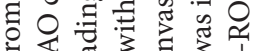

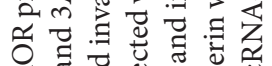

워

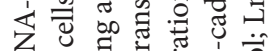

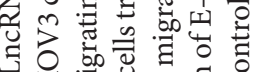

త.

ज.

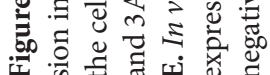


pointed that the location of LncRNA-ROR was a binding site for core transcription factors (TFs) Oct4, Sox2, and Nanog; and it acted as a ceRNA (competing endogenouse RNA) to regulate the expression of those core TFs (Wang et al. 2013). "microRNA sponge", which acted as an artificial miRNA inhibitor that created a loss-of-function phenotype for an entire miRNA "seed" family (Ebert et al. 2007). LncRNA-ROR acted as a "microRNA sponge" and interacted with miR-145. Several studies have presented that LncRNA-ROR functions as a ceRNA when modulating concentration and biological functions of miR-145 (Zhou et al. 2014; Eades et al. 2015; Gao et al. 2016; Zou et al. 2016).

FLNB (Filamin B) is a cytoskeletal protein encoded by the gene on autosomal chromosome 3 . It is cross-linked with actin to form a three-dimensional cytoskeletal network and maintain cell morphology (Tsui et al. 2016; Devis et al. 2017). FLNB is involved in many physiological and biochemical activities, including transcriptional regulation and cell proliferation. Recent studies have shown that filament A and B play a key role in tumorigenesis, bone disease, cell migration and adhesion (Ravipaty et al. 2017; Ahmed et al. 2018). In our study, we identified FLNB as a target gene for miR-145 involved in the EMT process in ovarian cancer cells for the first time.

Currently, many lncRNAs, except LncRNA-ROR, have been reported as key regulators of the occurrence and development of ovarian cancer (Zhang et al. 2018). And LncRNA-ROR remains unknown in ovarian cancer progression and metastasis. In this study, we demonstrated that LncRNA-ROR expression was higher in ovarian cancer tissues than in normal ovarian tissues. Futhermore, LncRNAROR promoted EMT by the miR-145/FLNB regulatory axis in ovarian cancer, providing a potential therapeutic target for ovarian cancer.

\section{Materials and Methods}

\section{Cell lines and human tissue specimens}

Both SKOV 3 and $3 \mathrm{AO}$ cells are epithelial ovarian carcinoma cell lines. SKOV 3 was obtained from the Shanghai Cell Bank of Chinese Academy of Sciences (Shanghai, China), 3AO was from the Shandong Academy of Medical Sciences (Jinan, China). Cells were maintained in RPMI 1640 medium (Gibco-BRL, Gaithersburg, MD, USA) supplemented with $10 \%(\mathrm{v} / \mathrm{v})$ fetal bovine serum at $37^{\circ} \mathrm{C}$ under a humidified $5 \%$ $\mathrm{CO}_{2}$ atmosphere. Human ovarian cancer tissue samples and normal ovarian tissue samples were collected from patients at The First Affiliated Hospital of Xi'an Jiaotong University, PR China. This study was approved by the Ethics Committee of The First Affiliated Hospital of Xi'an Jiaotong University,
China. Written consent was obtained from each study participant enrolled.

\section{Cell transfection}

pcDNA3.1/ROR (pcDNA3.1 as control), sh-ROR (shRNAnegative control as control) were purchased from Applied Biological Materials (ABM, Canada). The miR-145 mimics and control mimics were purchased from RiboBio (Guangzhou, China). Transfections were performed using the Lipofectamine 2000 kit (Invitrogen) according to the manufacturer's instructions.

\section{Quantitative real-time PCR ( $q R T-P C R)$}

Total RNA from tissues and cells was isolated with Trizol reagent (Invitrogen, CA, USA) according to the manufacturer's instructions. Reverse transcription was performed with PrimeScript RT reagent Kit (Takara, Japan) according to the manufacturer's protocol. Quantitative real-time PCR was performed using a SYBR Premix Ex Taq ${ }^{\text {TM }}$ II kit (Takara, Dalian, China) on a CFX96 real-time PCR system (Bio- Rad, Hercules, CA, USA) based on the manufacturer's instructions. The LncRNA-ROR and miR-145 level was calculated with the $2^{-\Delta \Delta \mathrm{Ct}}$ method, which was normalized to GAPDH and U6 mRNA, respectively. All assays were performed in triplicate. The following primer sequences were used: E-cadherin forward: 5'-GCTGCTCTTGCTGTTTCTTCG-3'; E-cadherin reverse: 5' -CCGCCTCCTTCTTCATCATAG-3'; FLNB forward: 5'-GGTGCTCAGCCAGAAGCGCA -3'; FLNB reverse: 5' -CAGGGCTCCCAGGGCTTTGC-3'; N-cadherin forward: 5' -GCCCCTCAAGTGTTACCTCAA-3'; N-cadherin reverse: 5'- AGCCGAGTGATGGTCCAATTT-3'; vimentin forward: 5'- AAGTTTGCTGACCTCTCTGAGGCT-3'; vimentin reverse: 5'- CTTCCATTTCACGCATCTGGCGTT-3'; $\beta$-actin forward :5' - TCCCTGGAGAAGAGCTACGA-3'; $\beta$-actin reverse :5'- AGCACTGTGTTGGCGTACAG-3'.

\section{Western blot}

Total protein was collected from cells by RIPA lysis buffer containing protease inhibitors (Roche, Indianapolis, IN, USA) and $1 \mathrm{mM}$ PMSF on ice. Protein concentration was measured using the BCA-200 Protein Assay kit (Pierce, Rockford, IL, USA). After heat denaturation at $100^{\circ} \mathrm{C}$ for $5 \mathrm{~min}$, proteins were separated by electrophoresis on $10 \%$ SDS-PAGE gels and then transferred onto nitrocellulose membranes (Pall Life Science, NY, USA). The membranes were blocked with $5 \%$ non-fat milk at room temperature for $1 \mathrm{~h}$, and then incubated overnight at $4^{\circ} \mathrm{C}$ with E-cadherin (1:1000, Cell Signaling Technology, Danvers, MA, USA), N-cadherin (1:1000, Cell Signaling Technology, Danvers, MA, USA), vimentin (1:500, Cell Signaling Technology, 
Danvers, MA, USA), FLNB (1:500, Abcam, Cambridge, MA, USA) and mouse anti-human $\beta$-actin (1:1000, Cell Signaling Technology, Danvers, MA, USA). After washing with TBST (Tris-buffered saline Tween-20), the blots were incubated with horse radish peroxidase (HRP)-conjugated goat antirabbit or anti-mouse IgG. Blots were visualized using ECL reagents (Pierce, Rockford, IL, USA) by a chemiluminescence imaging system (Bio-Rad, Richmond, CA, USA).

\section{Cell migration and invasion assay}

Cells were trypsinized and counted. A total of $1 \times 10^{5}$ cells (for migration assay) or $5 \times 10^{5}$ cells (for invasion assay) in $100 \mu \mathrm{l}$ serum-free medium were added into millicells (Millipore Co, Bedford, MA, USA) without (for migration assay) or with (for invasion assay) Matrigel (Becton Dickinson Labware, Bedford, MA, USA) coated. $500 \mu \mathrm{l}$ of 1640 medium containing $20 \%$ newborn bovine serum was added to the bottom chambers as the chemotactic factor. After incubation for $24 \mathrm{~h}$ (for migration assay) or $48 \mathrm{~h}$ (for invasion assay) at $37^{\circ} \mathrm{C}$. Migratory (or invasive) cells were counted and averaged from images of five random fields (original magnification $\times 200$ ) captured using an inverted light microscope. Each cell count was performed by three researchers.

\section{Luciferase reporter assay}

Cells were co-transfected with pRL-TK vector $(20 \mathrm{ng})$, wild-type or mutant reporter vectors (180 ng), along with miR-145 mimic or negative control at a final concentration
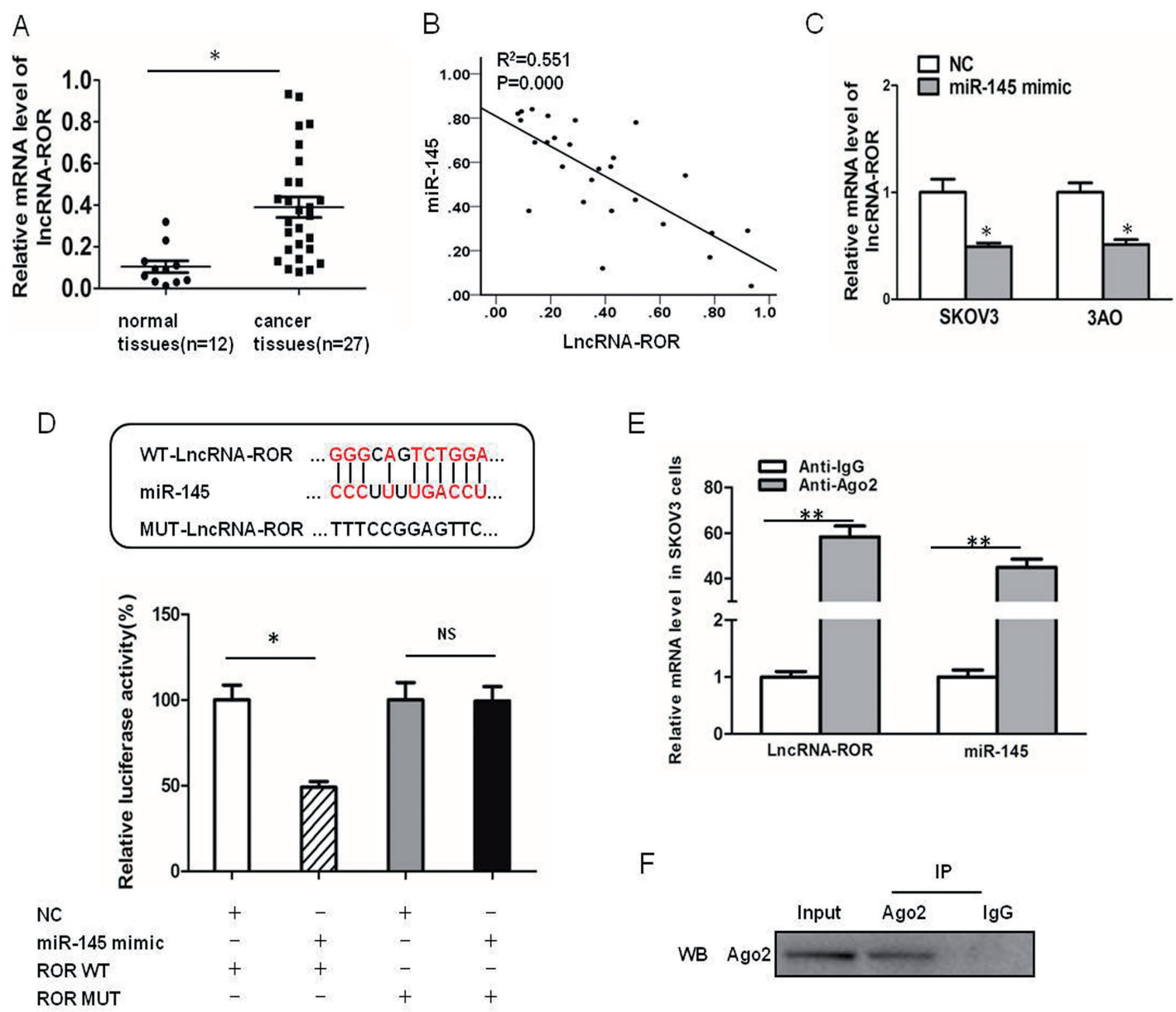

Figure 2. Reciprocal repression of lncRNA-ROR and miR-145 in ovarian cancer cells. A. The level of LncRNA-ROR expression in ovarian cancer tissues was significantly higher than in normal ovarian tissues. B. Scatter diagram showing LncRNA-ROR expression and miR145 expressions by qPCR and their correlations $\left(\mathrm{R}^{2}=0.551, \mathrm{P}=0.000\right)$ in 27 tissues of EOC. C. Overexpression of miR-145 inhibited LncRNA-ROR expression. D. The luciferase reporter assay showed that ROR MUT could bind to miR-145. E. RNA immunoprecipitation (RIP) experiments indicated that LncRNA-ROR and miR-145 could be enriched by Ago2 antibody. F. Western blot was used to detect the efficiency of immunoprecipitation reaction. ${ }^{*} p<0.05,{ }^{* *} p<0.01$. NC, negative control; ROR WT, wild-type vectors; ROR MUT, mutant-type vectors. 

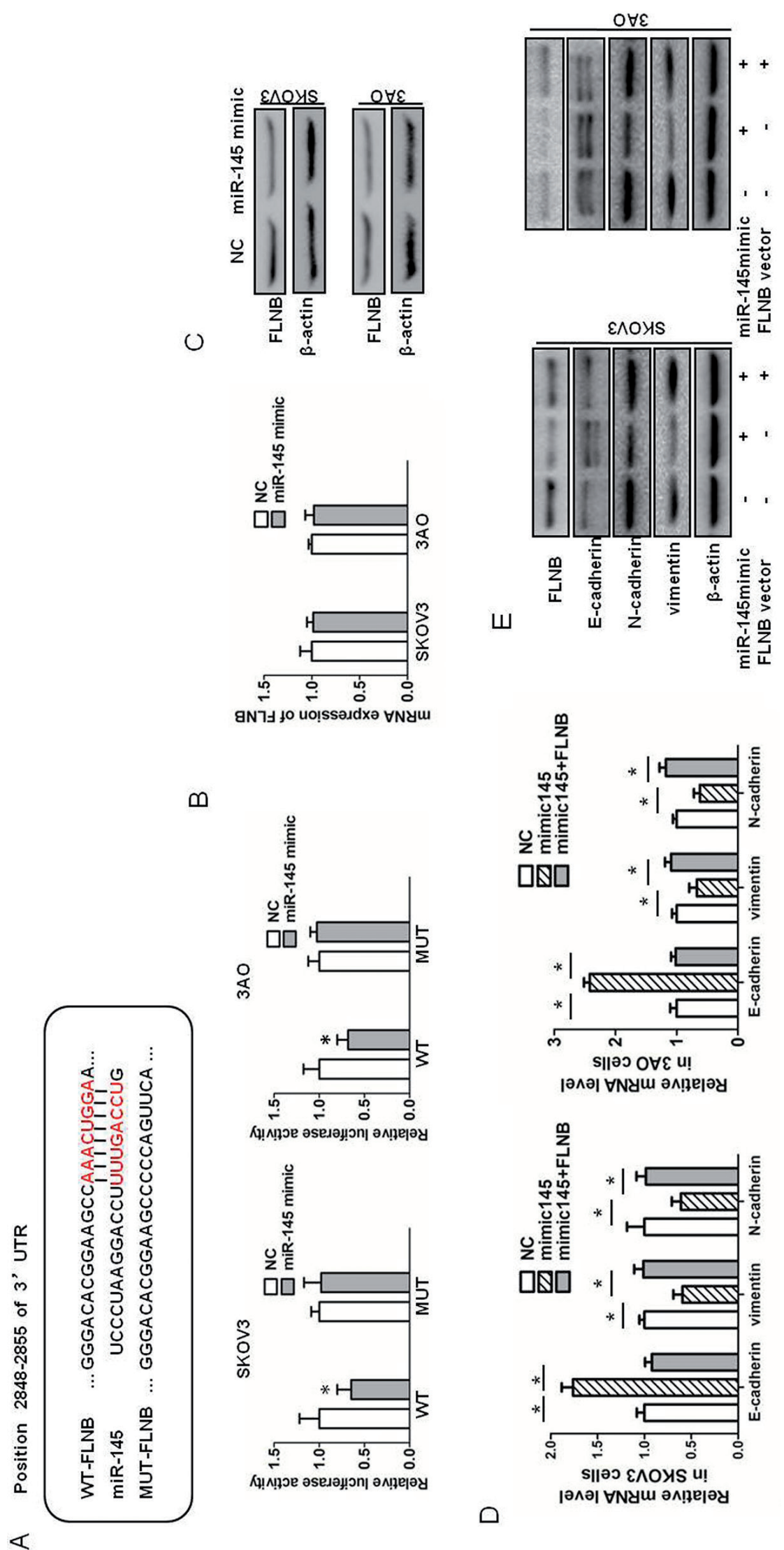


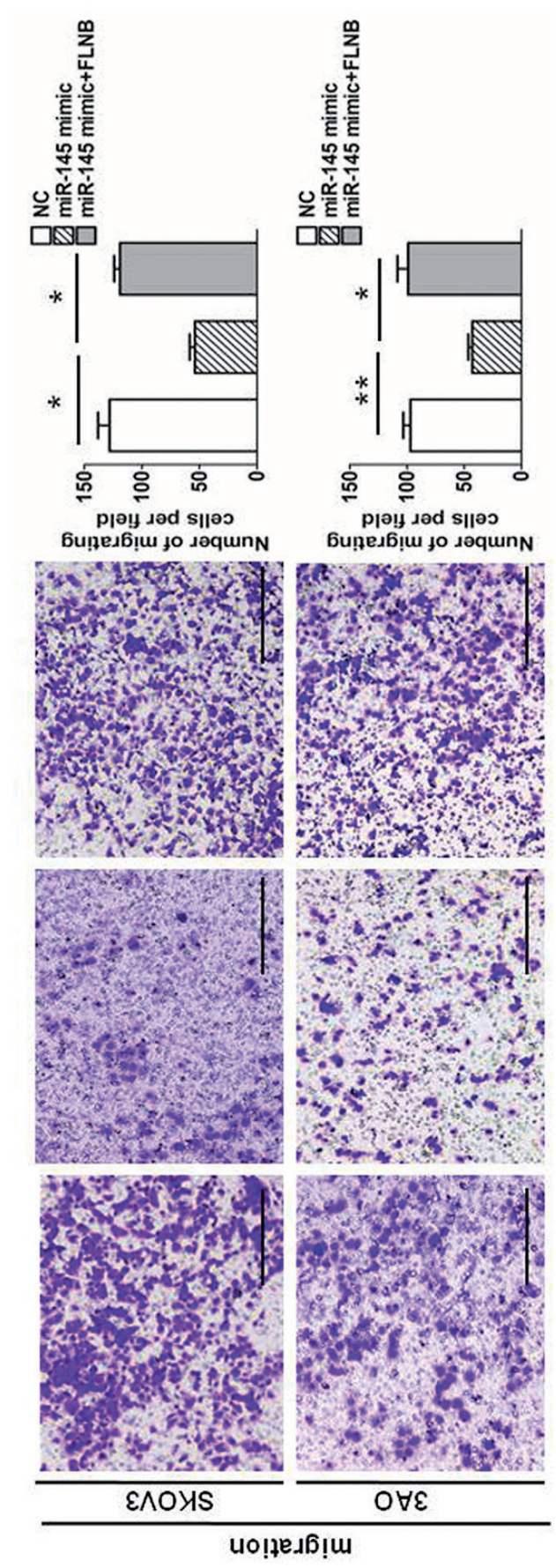

レ

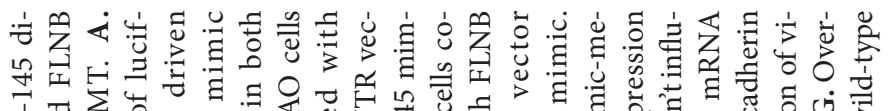

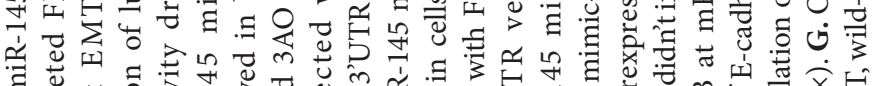

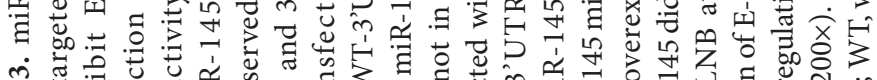

м.

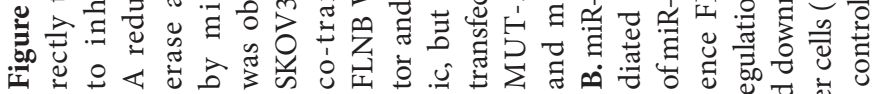

空芯芯芯

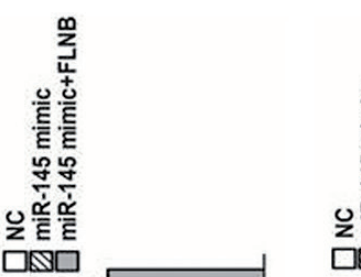

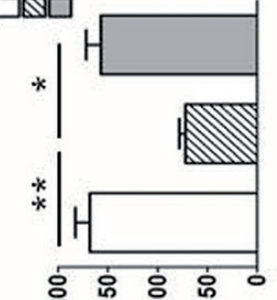

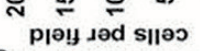
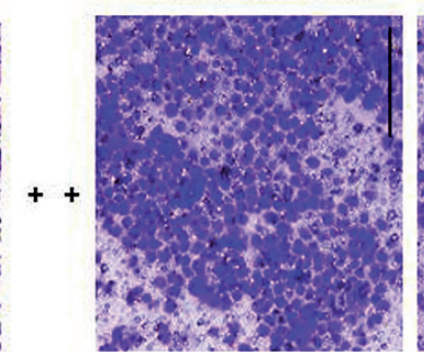

$*$

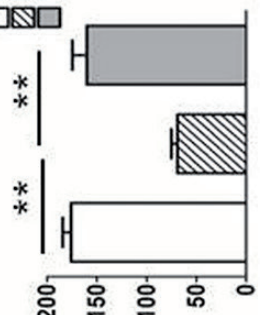

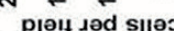
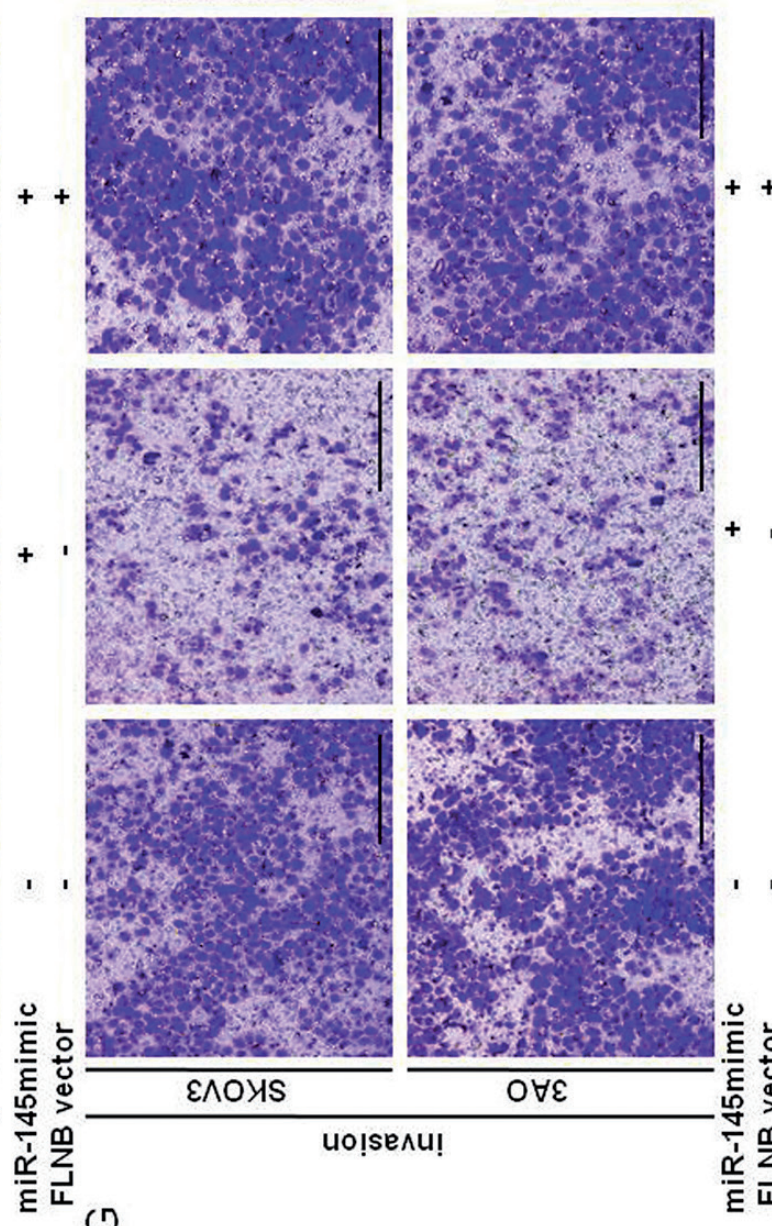

Gu!penu! to soquinN

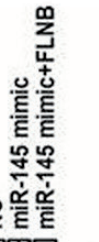

Gu!penu! to dəquinN

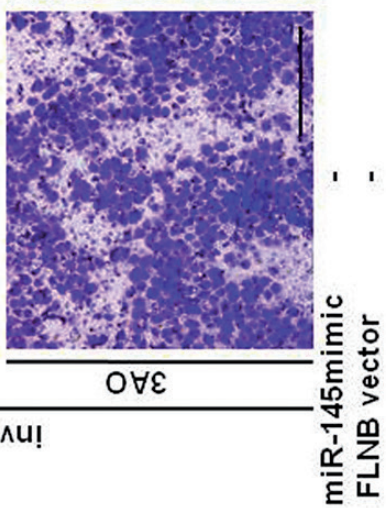

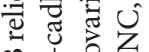

岩出毛自

在守言

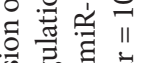

क

产 章

词范

ठ․ํ요

Q $)$

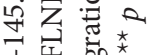

艄岁苔

洁

过

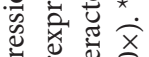

क्षे

○े

के 따 चै

可密

चु द्य

记

通表

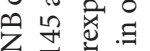

空要

च

要

苛苛

क ज्ञ

क०

卷

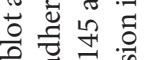

击

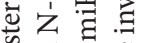

范范合

ن. $\Xi \vec{\Psi} \nabla$

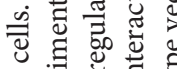

安范吉言

政

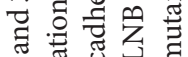

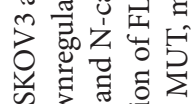

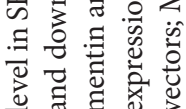

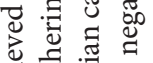


of $20 \mathrm{nM}$ using the X-treme GENE siRNA Transfection Reagent. $24 \mathrm{~h}$ after transfection, the relative firefly luciferase activity (normalized to Renilla luciferase activity) was measured using a dual-luciferase reporter gene assay system (Promega, Madison, WI, USA), and results were depicted as the percentage change over the respective control. Each experiment was performed in triplicate.

\section{RNA immunoprecipitation}

RNA immunoprecipitation (RIP) assays were performed using the EZMagna RIP kit (Millipore, Billerica, MA, USA) and the Ago 2 antibody (Millipore, Billerica, MA, USA) following the manufacturer's protocol. qRT-PCR analysis was performed to measure the expression levels of LncRNA-ROR and miR-145. Normal mouse IgG (Millipore, Billerica, MA, USA) was used as negative control.

\section{Statistical analysis}

All experiments were performed at least in triplicate, and each experiment was independently performed at least 3 times. The data was analyzed using SPSS 22.0 software (Chicago, IL, USA). Statistical differences were tested by Chisquare test or two-tailed $t$-test. Differences were considered significant at $p<0.05$ or highly significant at $p<0.001$. The error bar in figures stand for SD.

\section{Results}

LncRNA-ROR promoted migratory and invasive capacities and enhanced EMT of ovarian cancer cells

To investigate the function of LncRNA-ROR in ovarian cancer, we performed the migration /invasion assays in SKOV3 and

Table 1. Clinicopathological correlation of LncRNA-ROR to ovarian cancer

\begin{tabular}{llcc}
\hline Characteristics & & $n$ & $\begin{array}{c}\text { Relative mRNA level of } \\
\text { LncRNA-ROR }\end{array}$ \\
\hline Age $^{\mathrm{a}}$ & $<50$ & 12 & $0.451 \pm 0.217^{1}$ \\
& $\geq 50$ & 15 & $0.475 \pm 0.210$ \\
\cline { 2 - 4 } Clinical stages $^{\mathrm{a}}$ & I-II & 9 & $0.316 \pm 0.142^{2}$ \\
& III-IV & 18 & $0.499 \pm 0.204$ \\
\cline { 2 - 4 } Grade $^{\mathrm{b}}$ & G1 & 7 & $0.253 \pm 0.126^{3}$ \\
& G2 & 11 & $0.429 \pm 0.162$ \\
\hline
\end{tabular}

Data are mean \pm SD. $n$, number of cases; ${ }^{\text {a }}$ Fisher's Exact Test (twotailed); ${ }^{\mathrm{b}}$ one-way ANOVA (two-tailed); ${ }^{1} p=0.775,{ }^{2} p=0.025$, ${ }^{3} p=0.000$.
3AO cells. The result demonstrated that overexpression of LncRNA-ROR (Fig. 1A) promoted migration and invasion of SKOV 3 cells and $3 \mathrm{AO}$ cells (Fig. 1B), and down-regulated LncRNA-ROR (Fig. 1D) could significant inhibit cells migration/invasion capability (Fig. 1E). Western blot assays showed that high LncRNA-ROR could induce EMT (Fig. 1C) and down-expression of LncRNA-ROR reversed EMT (Fig. 1F).

Reciprocal repression of $\operatorname{lnc} R N A-R O R$ and miR-145 in ovarian cancer cells

We detected the expression of LncRNA-ROR and miR-145 in normal ovarian tissues $(n=12)$ and epithelial ovarian cancer tissues $(n=27)$, the results indicated that the expression of LncRNA-ROR in ovarian cancer tissues was significantly higher than that in normal ovarian tissues (Fig. 2A). In addition, the expression of LncRNA-ROR is inversely correlated with the expression of miR-145 in epithelial ovarian cancer tissues (Fig. 2B). Moreover, we found LncRNA-ROR level was positively associated with clinical stages and the grade of differentiation of malignant cells (Table 1).

Recently, lncRNAs have been identified as ceRNAs by interacting with miRNAs. Furthermore, miRNAs also negatively regulate lncRNAs expression by binding with lncRNAs. We used an miR-145 mimic to observe the alteration of LncRNA-ROR expression in ovarian cancer cells. miR-145 mimic could significantly decrease LncRNA-ROR expression (Fig. 2C). The interactions between miRNA and miRNA response ele-ments (MREs) inhibit the activity and expression of RNA molecules (Su et al. 2013). Furthermore, we used RNAHybrid software to predict that LncRNA-ROR had MRE directly combined with miR-145 seed sequence, suggested that LncRNA-ROR could be a ceRNA which sponges miR-145. The luciferase reporter assay showed that transfection of miR-145 significantly inhibited the luciferase activity in cells transfected with ROR WT (wild-type vectors), while no change of luciferase activity was found in ROR MUT (mutant-type vectors vectors) transfected cells (Fig. 2D). RIP experiments indicated that Ago2 antibody enriched more LncRNA-ROR and miR-145 than that of IgG antibody (Fig. 2E, F). The results suggested that LncRNA-ROR had ceRNA function and could competently bind to miR-145. Therefore, we concluded that LncRNA-ROR and miR-145 form a reciprocal repression regulatory loop to regulate ovarian cancer progression.

\section{miR-145 directly targeted FLNB to inhibit EMT}

We predicted putative target genes of miR-145 by searching the TargetScan database (release 5.1, http://www.targetscan.org/), and FLNB was chosen to be experimentally verified. Recent studies have shown that FLNB play a key role in tumorigenesis, 
but there were few studies on the biological function of FLNB in ovarian cancer, so we chose to focus on FLNB. The 3'-UTRs region of FLNB containing the wild-type or mutant putative miR-145 binding sites were cloned into a luciferase reporter plasmid. The luciferase reporter assay showed that luciferase activity was significantly inhibited in cells co-transfected with miR-145 mimic and FLNB WT-3' UTR vector, while no changes of luciferase activity were detected in cells transfected with miR-145 mimic and luciferase reporter plasmids containing mutant seeding sequence (Fig. 3A). Additionally, the result showed that only protein level of FLNB was lessened by miR145 (Fig. 3B, C). Overexpression of FLNB could counteract the blocking effect of miR-145 on EMT (Fig. 3D, E). In the same way, we found overexpression of FLNB could attenuate the inhibition of migration (Fig. 3F) and invasion (Fig. 3G) of ovarian cancer cells by miR-145. These data indicated the direct suppressive effect of miR-145 on FLNB expression.

\section{LncRNA-ROR regulated FLNB by miR-145}

In our study, we found LncRNA-ROR increased FLNB expression and overexpression of miR-145 attenuated the promotion of LncRNA-ROR on FLNB (Fig. 4A). Collectively, these results revealed that LncRNA-ROR promoted EMT through miR-145/FLNB pathways (Fig. 4B).

\section{Discussion}

LncRNA-ROR is a representative lncRNA that plays an important regulatory role in human physiological and pathological processes. In recent years, there has been an increasing amount of study about LncRNA-ROR could enhance the malignant of numerous tumors though promote cell reprogramming and the chemoresistance of several cancers (Li et al. 2016; Rezaei et al. 2016).
EMT can be described as a process that produces a complete loss of epithelial traits with the acquisition of mesenchymal traits (Hay 2005). During the last two decades, the EMT process has been regarded as the significant initial step and the underlying process in the tumor metastasis process (Fidler 1978; Birchmeier and Behrens 1994). Our study certified that overexpression of LncRNA-ROR promoted migration and invasion by cell migration and invasion assay. By Western blot assays, we found that high LncRNA-ROR could induce EMT and down-expression of LncRNA-ROR reversed EMT. The results consistent with previous studies (Fu et al. 2017; Li et al. 2017a). In this study, we prove that LncRNA-ROR is a positive regulator of EMT.

MiRNAs are small noncoding RNAs of 20-24-nucleotide long which conduce to the post-transcriptional regulation of gene expression. They take part in different biological processes, such as cell differentiation, proliferation, apoptosis (Bartel 2004). Since raised miRNAs involved in cancer formation, plenty of evidences manifest that miRNAs misexpression has been linked to cancer (Calin et al. 2002; He et al. 2005). The interactions between miRNA and MREs inhibit the activity and expression of RNA molecules (Su et al. 2013). RNA molecules, including lncRNA, compete with corresponding miRNA through common MREs and they act as reciprocal ceRNA (Sardina et al. 2017). Our research showed that LncRNA-ROR had MRE directly combined with miR-145 seed sequence. It indicated that LncRNAROR acts as a ceRNA which sponges miR-145 in ovarian cancer tissues. At the same time, we found overexpression of miR-145 inhibited LncRNA-ROR expression, but the molecular mechanism still needs to be further studied. Taken together, our results uncovered that oncogenic LncRNAROR and tumor suppressive miR-145 forms a reciprocal repression regulatory loop to promote ovarian cancer cell EMT (Fig. 4B).
A
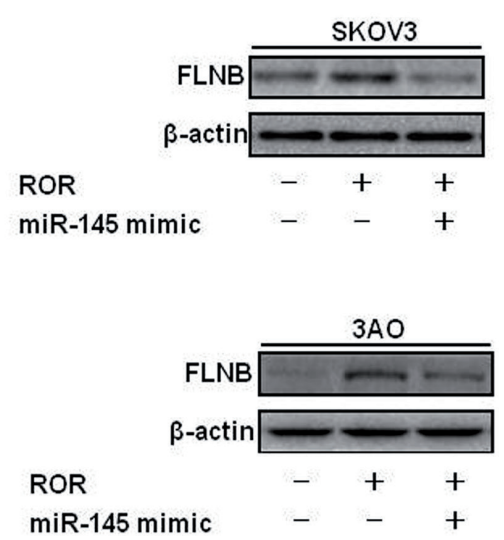

B

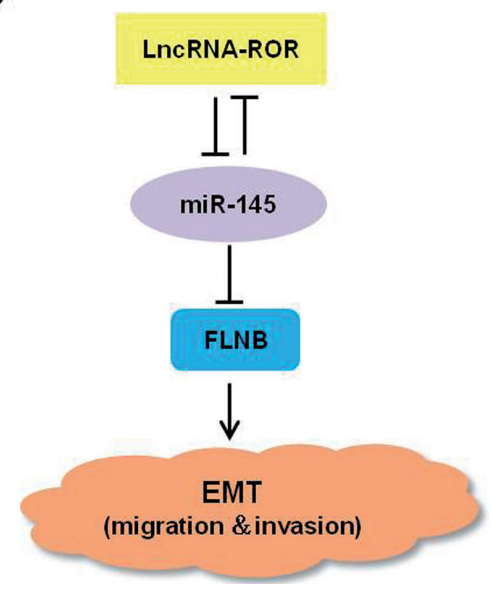

Figure 4. LncRNA-ROR regulated FLNB by miR-145. A. Western blot showed LncRNA-ROR increased FLNB expression and overexpression of miR-145 attenuated the promotion of LncRNAROR on FLNB. B. Schematic representation of the proposed mechanism of LncRNA-ROR mediated ovarian cancer progression through the miR-145/ FLNB pathway. FLNB, Filamin B; EMT, epithelial-mesenchymal transition. 
miR-145 blocked EMT by targeting FLNB. So far, these was only one research reported that FLNB promotes EMT in breast cancer ( $\mathrm{Li}$ et al. 2018). The relationship between miR-145 and FLNB in EMT process has not been reported.

To summarize, we affirm that LncRNA-ROR correlate with the occurrence and development of ovarian cancer. Through the LncRNA-ROR/miR-145/FLNB pathway, LncRNA-ROR promotes the invasion and migration of ovarian cancer by inducing EMT. Therefore, LncRNAROR is likely a biomarker of ovarian cancer and might be a candidate therapeutics for ovarian cancer therapy. In our study, we have only carried out experiments at the tissue and cytological level, and animal experiments will continue to confirm the results in vivo. We will explore in other tumors to see if LncRNA-ROR and miR-145 have the same regulatory mechanism in ovarian cancer.

Acknowledgement. This work was supported by National Natural Science Foundation of China (No.81702577), and Natural Science Basic Research Program of Shaanxi (No. 2018JQ8035), and the Fundamental Research Funds for the Central Universities (No. XZY012019105).

\section{References}

Ahmed W, Malik MFA, Saeed M, Haq F (2018): Copy number profiling of Oncotype DX genes reveals association with survival of breast cancer patients. Mol. Biol. Rep. 45, 2185-2192 https://doi.org/10.1007/s11033-018-4379-1

Arunkumar G, Deva Magendhra Rao AK, Manikandan M, Arun K, Vinothkumar V, Revathidevi S, Rajkumar KS, Rajaraman R, Munirajan AK (2017): Expression profiling of long non-coding RNA identifies linc-RoR as a prognostic biomarker in oral cancer. Tumour Biol. 39, 1010428317698366 https://doi.org/10.1177/1010428317698366

Bartel DP (2004): MicroRNAs: genomics, biogenesis, mechanism, and function. Cell 116, 281-297 https://doi.org/10.1016/S0092-8674(04)00045-5

Birchmeier W, Behrens J (1994): Cadherin expression in carcinomas: role in the formation of cell junctions and the prevention of invasiveness. Biochim. Biophys. Acta 1198, 11-26 https://doi.org/10.1016/0304-419X(94)90003-5

Calin GA, Dumitru CD, Shimizu M, Bichi R, Zupo S, Noch E, Aldler H, Rattan S, Keating M, Rai K, et al. (2002): Frequent deletions and down-regulation of micro- RNA genes miR15 and miR16 at 13q14 in chronic lymphocytic leukemia. Proc. Natl. Acad. Sci. USA 99, 15524-15529 https://doi.org/10.1073/pnas.242606799

Chen Y, Peng Y, Xu Z, Ge B, Xiang X, Zhang T, Gao L, Shi H, Wang C, Huang J (2017): LncROR promotes bladder cancer cell proliferation, migration, and epithelial-mesenchymal transition. Cell. Physiol. Biochem. 41, 2399-2410 https://doi.org/10.1159/000475910

Cheng EC, Lin H (2013): Repressing the repressor: a lincRNA as a MicroRNA sponge in embryonic stem cell self-renewal. Dev. Cell. 25, 1-2 https://doi.org/10.1016/j.devcel.2013.03.020

Devis L, Moiola CP, Masia N, Martinez-Garcia E, Santacana M, Stirbat TV, Brochard-Wyart F, Garcia A, Alameda F, Cabrera S, et al. (2017): Activated leukocyte cell adhesion molecule (ALCAM) is a marker of recurrence and promotes cell migration, invasion, and metastasis in early-stage endometrioid endometrial cancer. J. Pathol. 241, 475-487 https://doi.org/10.1002/path.4851

Eades G, Wolfson B, Zhang Y, Li Q, Yao Y, Zhou Q (2015): lincRNARoR and miR-145 regulate invasion in triple-negative breast cancer via targeting ARF6. Mol. Cancer Res. 13, 330-338 https://doi.org/10.1158/1541-7786.MCR-14-0251

Ebert MS, Neilson JR, Sharp PA (2007): MicroRNA sponges: competitive inhibitors of small RNAs in mammalian cells. Nat. Methods 4, 721-726 https://doi.org/10.1038/nmeth1079

Fidler IJ (1978): Tumor heterogeneity and the biology of cancer invasion and metastasis. Cancer Res. 38, 2651-2660

Fu Z, Li G, Li Z, Wang Y, Zhao Y, Zheng S, Ye H, Luo Y, Zhao X, Wei L, et al. (2017): Endogenous miRNA Sponge LincRNA-ROR promotes proliferation, invasion and stem cell-like phenotype of pancreatic cancer cells. Cell Death Discov. 3, 17004 https://doi.org/10.1038/cddiscovery.2017.4

Gao S, Wang P, Hua Y, Xi H, Meng Z, Liu T, Chen Z, Liu L (2016): ROR functions as a ceRNA to regulate Nanog expression by sponging miR-145 and predicts poor prognosis in pancreatic cancer. Oncotarget 7, 1608-1618 https://doi.org/10.18632/oncotarget.6450

Guttman M, Rinn JL (2012): Modular regulatory principles of large non-coding RNAs. Nature 482, 339-346 https://doi.org/10.1038/nature10887

Hay ED (2005): The mesenchymal cell, its role in the embryo, and the remarkable signaling mechanisms that create it. Dev. Dyn. 233, 706-720 https://doi.org/10.1002/dvdy.20345

He L, Thomson JM, Hemann MT, Hernando-Monge E, Mu D, Goodson S, Powers S, Cordon-Cardo C, Lowe SW, Hannon GJ, Hammond SM (2005): A microRNA polycistron as a potential human oncogene. Nature 435, 828-833 https://doi.org/10.1038/nature03552

Jiang HH, Lou YH, Wang XY, Han Y, Cui ZM (2016): Expression and function of long intergenic non-protein coding RNAregulator of reprogramming in high-grade ovarian serous cancer. Zhonghua Fu Chan Ke Za Zhi 51, 921-927 (in Chinese)

Li C, Lu L, Feng B, Zhang K, Han S, Hou D, Chen L, Chu X, Wang $\mathrm{R}$ (2017a): The lincRNA-ROR/miR-145 axis promotes invasion and metastasis in hepatocellular carcinoma via induction of epithelial-mesenchymal transition by targeting ZEB2. Sci. Rep. 7, 4637 https://doi.org/10.1038/s41598-017-04113-w

Li H, Jiang X, Niu X (2017b): Long non-coding RNA reprogramming (ROR) promotes cell proliferation in colorectal cancer via affecting P53. Med. Sci. Monit. 23, 919-928 https://doi.org/10.12659/MSM.903462

Li J, Choi PS, Chaffer CL, Labella K, Hwang JH, Giacomelli AO, Kim JW, Ilic N, Doench JG, Ly SH, et al. (2018): An alternative splicing switch in FLNB promotes the mesenchymal cell state in human breast cancer. Elife 7, e37184 
https://doi.org/10.7554/eLife.37184

Li L, Gu M, You B, Shi S, Shan Y, Bao L, You Y (2016): Long noncoding RNA ROR promotes proliferation, migration and chemoresistance of nasopharyngeal carcinoma. Cancer Sci. 107, 1215-1222 https://doi.org/10.1111/cas.12989

Liu T, Chi H, Chen J, Chen C, Huang Y, Xi H, Xue J, Si Y (2017): Curcumin suppresses proliferation and in vitro invasion of human prostate cancer stem cells by ceRNA effect of miR-145 and IncRNA-ROR. Gene 631, 29-38 https://doi.org/10.1016/j.gene.2017.08.008

Loewer S, Cabili MN, Guttman M, Loh YH, Thomas K, Park IH, Garber M, Curran M, Onder T, Agarwal S, et al. (2010): Large intergenic non-coding RNA-RoR modulates reprogramming of human induced pluripotent stem cells. Nat. Genet. 42, 1113-1117 https://doi.org/10.1038/ng.710

Qu CH, Sun QY, Zhang FM, Jia YM (2017): Long non-coding RNA ROR is a novel prognosis factor associated with non-small-cell lung cancer progression. Eur. Rev. Med. Pharmacol. Sci. 21, 4087-4091

Rauh-Hain JA, Birrer M, Del Carmen MG (2016): Carcinosarcoma of the ovary, fallopian tube, and peritoneum: Prognostic factors and treatment modalities. Gynecol. Oncol. 142, 248-254 https://doi.org/10.1016/j.ygyno.2016.06.003

Ravipaty S, Wu W, Dalvi A, Tanna N, Andreazi J, Friss T, Klotz A, Liao C, Garren J, Schofield S, et al. (2017): Clinical validation of a serum protein panel (FLNA, FLNB and KRT19) for diagnosis of prostate cancer. J. Mol. Biomark. Diagn. 8, 323 https://doi.org/10.1158/1538-7445.AM2017-3826

Rezaei M, Emadi-Baygi M, Hoffmann MJ, Schulz WA, Nikpour P (2016): Altered expression of LINC-ROR in cancer cell lines and tissues. Tumour Biol. 37, 1763-1769 https://doi.org/10.1007/s13277-015-3933-x

Sardina DS, Alaimo S, Ferro A, Pulvirenti A. Giugno R (2017): A novel computational method for inferring competing endogenous interactions. Brief Bioinform. 18, 1071-1081 https://doi.org/10.1093/bib/bbw084

Shang M, Wang X, Zhang Y, Gao Z, Wang T, Liu R (2018): LincRNAROR promotes metastasis and invasion of esophageal squamous cell carcinoma by regulating miR-145/FSCN1. Onco Targets Ther. 11, 639-649 https://doi.org/10.2147/OTT.S157638

Su X, Xing J, Wang Z, Chen L, Cui M, Jiang B (2013): microRNAs and ceRNAs: RNA networks in pathogenesis of cancer. Chin. J. Cancer Res. 25, 235-239

Thiery JP (2003): Epithelial-mesenchymal transitions in development and pathologies. Curr. Opin. Cell. Biol. 15, 740-746 https://doi.org/10.1016/j.ceb.2003.10.006

Tripathi MK, Doxtater K, Keramatnia F, Zacheaus C, Yallapu MM, Jaggi M, Chauhan SC (2018): Role of lncRNAs in ovarian cancer: defining new biomarkers for therapeutic purposes. Drug Discov. Today 23, 1635-1643 https://doi.org/10.1016/j.drudis.2018.04.010
Tsui JC, Lau CP, Cheung AC, Wong KC, Huang L, Tsui SK, Kumta SM (2016): Differential expression of filamin B splice variants in giant cell tumor cells. Oncol. Rep. 36, 3181-3187 https://doi.org/10.3892/or.2016.5197

Wang L, Yang F, Jia LT, Yang AG (2017): missing links in epithelialmesenchymal transition: long non-coding RNAs enter the arena. Cell. Physiol. Biochem. 44, 1665-1680 https://doi.org/10.1159/000485766

Wang S, Liu F, Deng J, Cai X, Han J, Liu Q (2016a): Overexpression of LncRNA-ROR predicts a poor outcome in gallbladder cancer patients and promotes the tumor cells proliferation, migration, and invasion. Tumour Biol. 37, 12867-12875 https://doi.org/10.1007/s13277-016-5210-z

Wang SH, Zhang MD, Wu XC, Weng MZ, Zhou D, Quan ZW (2016b): Long noncoding RNA ROR regulates proliferation, invasion, and stemness of gastric cancer stem cell. Cell Reprogram. 18, 319-326 https://doi.org/10.1089/cell.2016.0001

Wang Y, Xu Z, Jiang J, Xu C, Kang J, Xiao L, Wu M, Xiong J, Guo X, Liu H (2013): Endogenous miRNA sponge lincRNA-RoR regulates Oct4, Nanog, and Sox2 in human embryonic stem cell self-renewal. Dev. Cell 25, 69-80 https://doi.org/10.1016/j.devcel.2013.03.002

Whitehead J, Pandey GK, Kanduri C (2009): Regulation of the mammalian epigenome by long noncoding RNAs. Biochim. Biophys. Acta 1790, 936-947 https://doi.org/10.1016/j.bbagen.2008.10.007

Wilusz JE, Sunwoo H, Spector DL (2009): Long noncoding RNAs: functional surprises from the RNA world. Genes Dev. 23, 1494-1504 https://doi.org/10.1101/gad.1800909

Zhang HY, Liang F, Zhang JW, Wang F, Wang L, Kang XG (2017): Effects of long noncoding RNA-ROR on tamoxifen resistance of breast cancer cells by regulating microRNA-205. Cancer Chemother. Pharmacol. 79, 327-337 https://doi.org/10.1007/s00280-016-3208-2

Zhang Y, Wang J, Wu D, Li M, Zhao F, Ren M, Cai Y, Dou J (2018): IL-21-secreting hUCMSCs combined with miR-200c inhibit tumor growth and metastasis via repression of Wnt/beta-catenin signaling and epithelial-mesenchymal transition in epithelial ovarian cancer. Onco Targets Ther. 11, 2037-2050 https://doi.org/10.2147/OTT.S147855

Zhou X, Gao Q, Wang J, Zhang X, Liu K, Duan Z (2014): Linc-RNARoR acts as a "sponge" against mediation of the differentiation of endometrial cancer stem cells by microRNA-145. Gynecol. Oncol. 133, 333-339

https://doi.org/10.1016/j.ygyno.2014.02.033

Zou G, Liu T, Guo L, Huang Y, Feng Y, Huang Q, Duan T (2016): miR-145 modulates lncRNA-ROR and Sox2 expression to maintain human amniotic epithelial stem cell pluripotency and beta islet-like cell differentiation efficiency. Gene 591, 48-57 https://doi.org/10.1016/j.gene.2016.06.047

Received: May 21, 2019

Final version accepted: July 28, 2019 